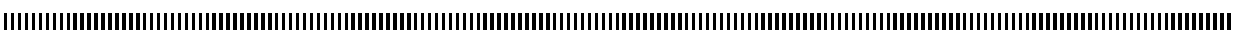

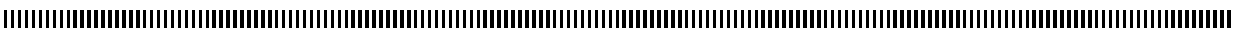

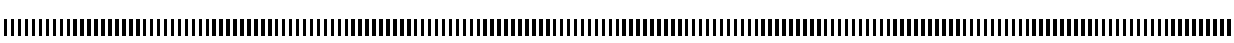

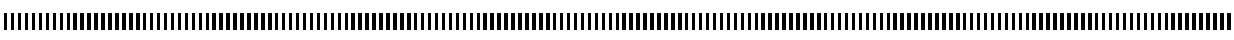

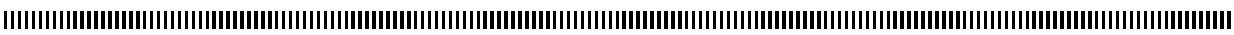

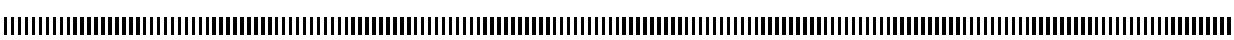

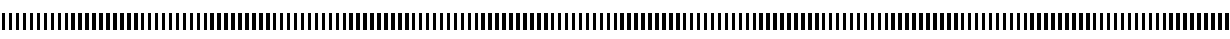

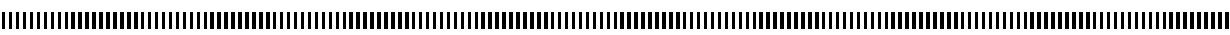

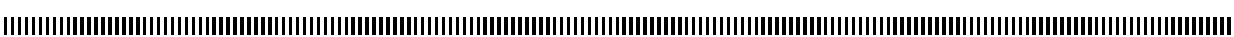
|||||||||||||||||||||||||||||||||||||||||||||||||||||||||||||||||||||||||||||||||||||||||||||||||||||||||||||||||||||||||||||||||||||||||||||||||||||||||||||||||||||||||||||||||||||||||||||||||||||

\title{
Some efficient methods for computing the determinant of large sparse matrices
}

\author{
E. KAMGNIA(1) - L. B. NGUENANG(1) \\ (1) Department of Computer Science, University of Yaounde I, Yaounde, Cameroon. \\ Emails: erkamgnia@yahoo.fr, lnguenang@yahoo.fr
}

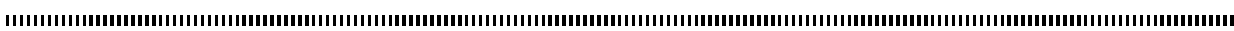

ABSTRACT. The computation of determinants intervenes in many scientific applications, as for example in the localization of eigenvalues of a given matrix $A$ in a domain of the complex plane. When a procedure based on the application of the residual theorem is used, the integration process leads to the evaluation of the principal argument of the complex logarithm of the function $g(z)=$ $\operatorname{det}((z+h) I-A) / \operatorname{det}(z I-A)$, and a large number of determinants is computed to insure that the same branch of the complex logarithm is followed during the integration. In this paper, we present some efficient methods for computing the determinant of a large sparse and block structured matrix. Tests conducted using randomly generated matrices show the efficiency and robustness of our methods.

RÉSUMÉ. Le calcul de déterminants intervient dans certaines applications scientifiques, comme par exemple dans le comptage du nombre de valeurs propres d'une matrice situées dans un domaine borné du plan complexe. Lorsqu'on utilise une approche fondée sur l'application du théorème des résidus, l'intégration nous ramène à l'évaluation de l'argument principal du logarithme complexe de la fonction $g(z)=\operatorname{det}((z+h) I-A) / \operatorname{det}(z I-A)$, en un grand nombre de points, pour ne pas sauter d'une branche à l'autre du logarithme complexe. Nous proposons dans cet article quelques méthodes efficaces pour le calcul du déterminant d'une matrice grande et creuse, et qui peut être transformée sous forme de blocs structurés. Les résultats numériques, issus de tests sur des matrices générées de façon aléatoire, confirment l'efficacité et la robustesse des méthodes proposées.

KEYWORDS : Determinant, eigenvalues, LU factorization, characteristic polynomial, Schur complement, SPIKE

MOTS-CLÉS : Déterminants, valeurs propres, polynôme caractéristique, factorisation LU, complément de Schur, SPIKE

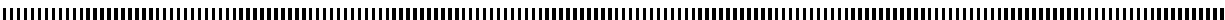




\section{Introduction}

The computation of determinants is needed in scientific applications. One such application is the localisation of eigenvalues of a given matrix $A \in \mathbb{R}^{n \times n}$ in a domain of the complex plaine. When one proceeds as in [2,3] by applying the residue theorem, the number $N_{\Gamma}$ of complex eigenvalues which are surrounded by a given curve $(\Gamma)$ is computed by evaluating the integral

$$
N_{\Gamma}=\frac{1}{2 i \pi} \int_{\Gamma} \frac{d}{d z} \log \operatorname{det}(z I-A) d z .
$$

The evaluation of this integral is also applied in works dealing with non-linear eigenvalue problems $[4,8]$. For instance, to compute the number $N_{\Gamma}$ of complex eigenvalues which are surrounded by a given curve $(\Gamma)$, the procedure EIGENCNT [3] implies evaluating the characteristic polynomial at many points of $(\Gamma)$ since the selected points must support the quadrature of

$$
N_{\Gamma}=\frac{1}{2 i \pi} \int_{\Gamma} \frac{f^{\prime}(z)}{f(z)} d z .
$$

Let $z$ and $z+h$ be two points of $(\Gamma)$. Since

$$
\begin{aligned}
(z+h) I-A & =(z I-A)+h I \\
& =(z I-A)(I+h R(z)),
\end{aligned}
$$

where $R(z)=(z I-A)^{-1}$, it follows that

$$
f(z+h)=f(z) \operatorname{det}(I+h R(z)) .
$$

Let $\Phi_{z}(h)=\operatorname{det}(I+h R(z)), g(z)=\frac{f^{\prime}(z)}{f(z)}$, then

$$
\begin{aligned}
\int_{z}^{z+h} \frac{f^{\prime}(z)}{f(z)} d z & =\log (f(z+h))-\log (f(z)) \\
& =\log \left(\frac{f(z+h)}{f(z)}\right) \\
& =\log \left(\Phi_{z}(h)\right) \\
& =\log \left|\Phi_{z}(h)\right|+i \arg \left(\Phi_{z}(h)\right)
\end{aligned}
$$

Given that this is a multivalued function, the goal is therefore to insure that a branch corresponding to a given determination of the complex logarithm can be followed while avoiding any jump to another determination. In [3], a stepsize control is introduced for insuring this property. For large matrices, the number of necessary determinant evaluations may become very high especially when many eigenvalues lie near the boundary $(\Gamma)$.

In this paper, we present some efficient techniques for computing the determinant of large matrices that can be put into block structured form. Such matrices arise in the discretization of partial differential equations especially in combination with domain decomposition and more generally from many sparse matrices after reordering, e.g. with the help of some graph partitioning tools see e.g. [1,6]. 
Computing a determinant is efficiently done through an LU-factorization of the matrix with the standard permutation strategies. When the matrix is sparse, the LU-factorization is either with row permutation $\mathrm{PA}=\mathrm{LU}[11,12]$ or with and additional column permutation PAQ=LU [10], where $P$ and $Q$ are permutation matrices, and where $L$ and $U$ are respectively a lower-triangular matrix with unit main diagonal and an upper-triangular matrix; then $\operatorname{det}(A)=\operatorname{det}(P) \operatorname{det}(U)$ or $\operatorname{det}(A)=\operatorname{det}(P) \operatorname{det}(U) \operatorname{det}(Q)$, where $\operatorname{det}(P)= \pm 1, \operatorname{det}(Q)= \pm 1$ and $\operatorname{det}(U)$ is the product of all the diagonal entries of $U$.

\section{Avoiding overflows and underflows when computing a determinant}

For any non singular matrix $A \in \mathbb{C}^{n \times n}$, let us consider its LU factorization $P A Q=$ $L U$ where $P$ and $Q$ are permutation matrices of signatures $\sigma_{P}$ and $\sigma_{Q}$. Then $\operatorname{det}(A)=$ $\sigma_{P} \sigma_{Q}\left(\prod_{i=1}^{n} u_{i i}\right)$ where $u_{i i} \in \mathbb{C}$ are the diagonal entries of $U$. When the matrix $A$ is not correctly scaled, the product $\left(\prod_{i=1}^{n} u_{i i}\right)$ may generate an overflow or underflow. To avoid this, we encode the determinant using the triplet $(\rho, K, n)$ so that

$$
\operatorname{det}(A)=\rho K^{n}
$$

where:

$$
\begin{gathered}
\rho=\sigma_{P} \sigma_{Q} \prod_{i=1}^{n} \frac{u_{i i}}{\left|u_{i i}\right|}, \quad(\rho \in \mathbb{C} \text { with }|\rho|=1), \\
K=\sqrt[n]{\prod_{i=1}^{n}\left|u_{i i}\right|}(K>0) .
\end{gathered}
$$

The quantity $K$ is computed through its logarithm:

$$
\log (K)=\frac{1}{n} \sum_{i=1}^{n} \log \left(\left|u_{i i}\right|\right) .
$$

In this way, the value of the determinant is safely computed even when the matrix is not properly scaled; i.e. diagonal elements of $U$ vary sharply in magnitude. Before raising to power $n$, and to protect from under- or overflow, the positive constant $K$ must be in the interval $\left[\frac{1}{\sqrt[n]{M_{f l}}}, \sqrt[n]{M_{f l}}\right]$ where $M_{f l}$ is the largest representable number in the underlying floating point system; otherwise the value of the determinant is not computed because it will lead to an overflow or underflow.

\section{Preliminary result}

If A can be put in the form $A=I+U V$, where $U \in \mathbb{C}^{m \times n}, V \in \mathbb{C}^{n \times m}$, then the following proposition shows how to efficiently compute the determinant of $A$.

Proposition 3.1 If $U \in \mathbb{C}^{m \times n}, V \in \mathbb{C}^{n \times m}$ then, $\operatorname{det}\left(I_{m}+U V\right)=\operatorname{det}\left(I_{n}+V U\right)$.

Note: this proposition appears as exercice 6.2.7 in [9]. 
Proof. Any eigenvalue of $U V$ is either zero or is an eigenvalue of $V U$. Indeed, let $\lambda$ be a nonzero eigenvalue of $U V$ and $w \neq 0$, an associated eigenvector: $(U V) w=\lambda w$. Therefore $(V U)(V w)=\lambda(V w)$. Let us prove by contradiction that $V w \neq 0$ : if $V w=0$, and since $U(V w)=\lambda w$, therefore $\lambda w=0$, and finally, $\lambda=0$.

Since $V w \neq 0$, it follows that $(\lambda, V w)$ is an eigenpair of $V U$; therefore the nonzero eigenvalues of $U V$ are nonzero eigenvalues of $V U$. Since $U$ and $V$ can be interchanged without affecting the result, the two matrices have the same nonzero eigenvalues. An argument of continuity proves that their algebraic multiplicities are the same. For any square matrix $M$, let us denote its spectrum by $\Lambda(M)$. It then follows that, $\Lambda(U V) \cup$ $\{0\}=\Lambda(V U) \cup\{0\}$, which implies $\Lambda\left(I_{m}+U V\right) \cup\{1\}=\Lambda\left(I_{n}+V U\right) \cup\{1\}$ and therefore, $\operatorname{det}\left(I_{m}+U V\right)=\operatorname{det}\left(I_{n}+V U\right)$.

This proposition is especially useful when, either $m \ll n$, or $n \ll m$, since it may drastically reduce the order of the matrix for which determinant is sought. We make use of this proposition in the following sections.

\section{Computing the determinant of a q-block tridiagonal matrix}

We now assume that the matrix $A$ is q-block tridiagonal:

$$
\left(\begin{array}{ccccc}
A_{1} & A_{1,2} & 0 & \ldots & 0 \\
A_{2,1} & A_{2} & \ddots & \ddots & \vdots \\
0 & \ddots & \ddots & \ddots & 0 \\
\vdots & \ddots & \ddots & \ddots & A_{q-1, q} \\
0 & \ldots & 0 & A_{q, q-1} & A_{q}
\end{array}\right)
$$

where for $i=1, \cdots, q-1$ the blocks $A_{i+1, i}$ and $A_{i, i+1}$ are corner matrices defined by:

$$
\begin{gathered}
A_{i, i+1}=\left(\begin{array}{cc}
0 & 0 \\
B_{i} & 0
\end{array}\right), \\
A_{i+1, i}=\left(\begin{array}{cc}
0 & C_{i+1} \\
0 & 0
\end{array}\right) ;
\end{gathered}
$$

with $A_{i} \in \mathbb{C}^{n_{i} \times n_{i}}, B_{i} \in \mathbb{C}^{b_{i} \times l_{i}}, C_{i+1} \in \mathbb{C}^{c_{i+1} \times r_{i+1}}$. We assume throughout that $c_{i} \leq n_{i}, b_{i} \leq n_{i}$ and $l_{i-1}+r_{i+1} \leq n_{i}$.

\subsection{Sequential method}

We begin with a block sequential algorithm with partial pivoting. From the LU factorization $P_{1} A_{1}=L_{1} U_{1}$ and by partitioning $\tilde{A}=P A$ where $P=\left(\begin{array}{cc}P_{1} & 0 \\ 0 & I\end{array}\right)$ and $A=\left(\begin{array}{cc}A_{1} & A_{12} \\ A_{21} & A_{22}\end{array}\right)$, then $\tilde{A}$ admits the following LU decomposition:

$$
\tilde{A}=\left(\begin{array}{cc}
I & 0 \\
A_{21}\left(P_{1} A_{1}\right)^{-1} & I
\end{array}\right)\left(\begin{array}{cc}
P_{1} A_{1} & P_{1} A_{12} \\
0 & S_{1}
\end{array}\right)
$$


with the Schur complement $S_{1}=A_{22}-A_{21} U_{1}^{-1} L_{1}^{-1} P_{1} A_{12}$. Thus

$$
\operatorname{det}(A)=\operatorname{det}\left(P_{1}\right) \operatorname{det}\left(A_{1}\right) \operatorname{det}\left(S_{1}\right) .
$$

Consider the block partition

$$
U_{1}^{-1}=\left[\begin{array}{cc}
\left(U_{1}^{-1}\right)^{11} & \left(U_{1}^{-1}\right)^{12} \\
0 & \left(U_{1}^{-1}\right)^{22}
\end{array}\right] \in \mathbb{C}^{n_{1} \times n_{1}},
$$

where $\left(U_{1}^{-1}\right)^{22} \in \mathbb{C}^{r_{2} \times r_{2}}$

Let

$$
X=C_{2}\left(U_{1}^{-1}\right)^{22} \in \mathbb{C}^{c_{2} \times r_{2}}
$$

and

$$
G=L_{1}^{-1} P_{1}\left[\begin{array}{c}
0 \\
B_{1}
\end{array}\right]=\left[\begin{array}{l}
G_{1} \\
G_{2}
\end{array}\right],
$$

with $G_{1} \in \mathbb{C}^{n_{1}-r_{2} \times l_{1}}$ and $G_{2} \in \mathbb{C}^{r_{2} \times l_{1}}$; thus $X G_{2} \in \mathbb{C}^{c_{2} \times l_{1}}$.

Then the Schur complement $S_{1}$ can be easily built by

$$
\begin{aligned}
S_{1} & =A_{22}-\left[\begin{array}{l}
I \\
0
\end{array}\right]\left[\begin{array}{ll}
0 & X
\end{array}\right] L_{1}^{-1} P_{1}\left[\begin{array}{l}
0 \\
B
\end{array}\right]\left[\begin{array}{ll}
I & 0
\end{array}\right], \\
& =A_{22}-\left[\begin{array}{l}
I \\
0
\end{array}\right]\left[\begin{array}{ll}
0 & X
\end{array}\right]\left[\begin{array}{l}
G_{1} \\
G_{2}
\end{array}\right]\left[\begin{array}{ll}
I & 0
\end{array}\right], \\
& =A_{22}-\left[\begin{array}{cc}
X G_{2} & 0 \\
0 & 0
\end{array}\right] .
\end{aligned}
$$

Thus, only the leading $c_{2} \times l_{1}$ block of $A_{2}$ is affected when $A_{22}$ is transformed into $S_{1}$. The computation of $X G_{2}$ requires solving $l_{1}$ triangular systems of order $n_{1}$ for $G, c_{2}$ triangular systems of order $r_{2}$, for $X$ and a $\left(c_{2} \times r_{2}\right) \times\left(r_{2} \times l_{1}\right)$ matrix multiplication for $X G_{2}$.

Let $\tilde{A}_{2}$ be the transformed block; this approach is then recursively applied to the trailing matrix

$$
A_{22}=\left[\begin{array}{ccccc}
\tilde{A}_{2} & A_{2,3} & 0 & \ldots & 0 \\
A_{3,2} & A_{3} & \ddots & \ddots & \vdots \\
0 & \ddots & \ddots & \ddots & 0 \\
\vdots & \ddots & \ddots & \ddots & A_{q-1, q} \\
0 & \ldots & 0 & A_{q, q-1} & A_{q}
\end{array}\right]
$$

after $A_{22}$ has been corrected. Repeating this process $(q-1)$ times we deduce

$$
\operatorname{det}(A)=\prod_{i=1}^{q} \operatorname{det}\left(P_{i}\right) \operatorname{det}\left(U_{i}\right) .
$$

Definition 4.1 The method based on the formula (9) is called q-block sequential method. 
It is worth pointing out that when the LU factorization is carried out with left and right permutation, i.e, when $P_{1} A_{1} Q_{1}=L U$, the same process applies. Indeed let $F=U_{1}^{-1} G$, where $G$ is given by equation (7) and let $Y=Q_{1} F=\left[\begin{array}{c}Y_{1} \\ Y_{2}\end{array}\right]$, with $Y_{1} \in \mathbb{C}^{n_{1}-r_{2} \times l_{1}}$ and $Y_{2} \in \mathbb{C}^{c_{2} \times l_{1}} ;$ thus $C_{2} Y_{2} \in \mathbb{C}^{c_{2} \times l_{1}}$ and

$$
S_{1}=A_{22}-\left[\begin{array}{cc}
C_{2} Y_{2} & 0 \\
0 & 0
\end{array}\right]
$$

Thus as is the previous case, only the leading $c_{2} \times l_{1}$ block of $A_{2}$ is affected when $A_{22}$ is transformed into $S_{1}$; but computing $C_{2} Y_{2}$ is more expensive than computing $X G_{2}$ in the previous case as $c_{2}$ triangular systems of order $r_{2}$ to be solved are replaced by $l_{1}$ triangular systems of order $n_{1}$.

\subsection{Parallel method}

We now describe a parallel method for computing the determinant; the corresponding parallel algorithm is sketched in Algorithm 1. Considering the SPIKE transformation [7], let $D=\operatorname{diag}\left(A_{1}, A_{2}, \cdots, A_{q}\right)$ be assumed to be nonsingular, and for $i=$ $1, \cdots, q$, let $P_{i} A_{i} Q_{i}=L_{i} U_{i}$ a sparse LU factorization of the diagonal blocks. Let $P=\operatorname{diag}\left(P_{1}, P_{2}, \cdots, P_{q}\right), Q=\operatorname{diag}\left(Q_{1}, Q_{2}, \cdots, Q_{q}\right.$ and

$$
S \stackrel{\text { def }}{=} D^{-1} A=I_{n}+T
$$

where

$$
\begin{aligned}
& I_{n}=\operatorname{diag}\left(I_{s_{1}}, I_{r_{2}}, I_{l_{1}}, I_{s_{2}}, I_{r_{3}}, I_{l_{2}} \cdots, I_{r_{q}}, I_{l_{q-1}}, I_{s_{q}}\right),
\end{aligned}
$$

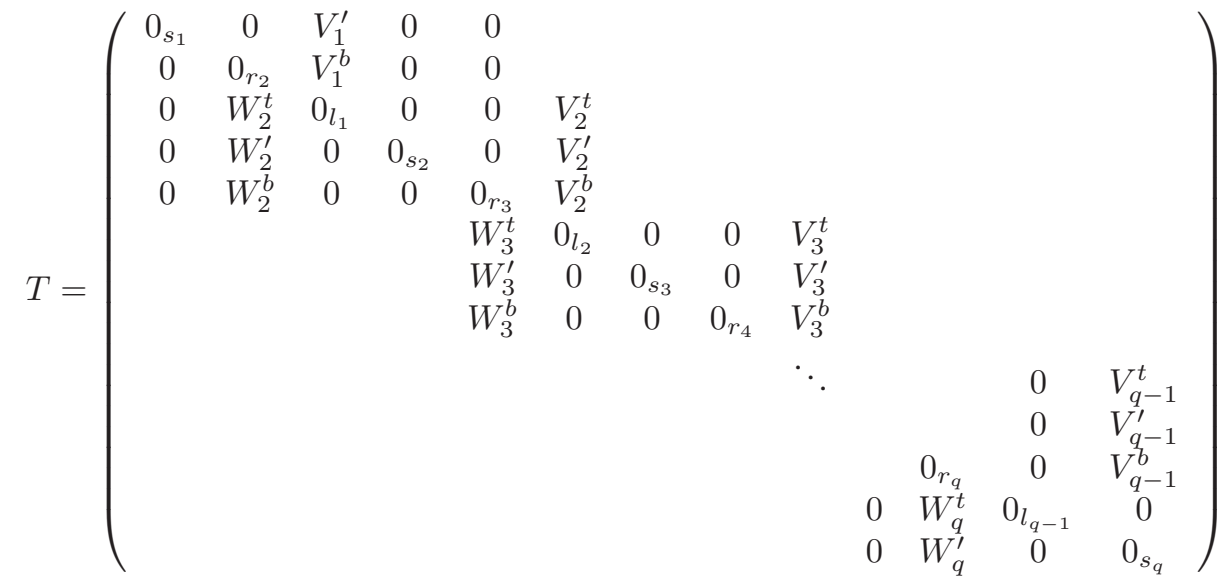

and where the block vectors are defined by:

$$
\begin{aligned}
& V_{1} \in \mathbb{C}^{n_{1} \times l_{1}} \equiv \mid \begin{array}{c|c}
V_{1}^{\prime} & s_{1} \\
V_{1}^{b} & r_{2},
\end{array} \\
& =\left(A_{1}\right)^{-1}\left(\begin{array}{c}
0 \\
B_{1}
\end{array}\right)=Q_{1} U_{1}^{-1} L_{1}^{-1} P_{1}\left(\begin{array}{c}
0 \\
B_{1}
\end{array}\right) \text {, }
\end{aligned}
$$


and for $i=2, \cdots, q-1$,

$$
\begin{aligned}
& V_{i} \in \mathbb{C}^{n_{i} \times l_{i}} \equiv \mid \begin{array}{c|c}
V_{i}^{t} & l_{i-1} \\
V_{i}^{\prime} & s_{i} \\
V_{i}^{b} & r_{i+1},
\end{array} \\
& =\left(A_{i}\right)^{-1}\left(\begin{array}{c}
0 \\
B_{i}
\end{array}\right)=Q_{i} U_{i}^{-1} L_{i}^{-1} P_{i}\left(\begin{array}{c}
0 \\
B_{i}
\end{array}\right) \text {, } \\
& W_{i} \in \mathbb{C}^{n_{i} \times r_{i}} \equiv \mid \begin{array}{c|c}
W_{i}^{t} & l_{i-1} \\
W_{i}^{\prime} & s_{i} \\
W_{i}^{b} & r_{i+1}
\end{array} \\
& =\left(A_{i}\right)^{-1}\left(\begin{array}{c}
C_{i} \\
0
\end{array}\right)=Q_{i} U_{i}^{-1} L_{i}^{-1} P_{i}\left(\begin{array}{c}
C_{i} \\
0
\end{array}\right) \text {, }
\end{aligned}
$$

and

$$
\begin{aligned}
& W_{q} \in \mathbb{C}^{n_{q} \times r_{q}} \equiv \begin{array}{c|c}
W_{q}^{t} & l_{q-1} \\
W_{q}^{\prime} & s_{q}
\end{array} \\
& =\left(A_{q}\right)^{-1}\left(\begin{array}{c}
C_{q} \\
0
\end{array}\right)=Q_{q} U_{q}^{-1} L_{q}^{-1} P_{q}\left(\begin{array}{c}
C_{q} \\
0
\end{array}\right) \text {. }
\end{aligned}
$$

These blocks are called spike vectors in [7]. With this partition, it is clear that:

$$
\operatorname{det}(A)=\left(\prod_{i=1}^{q} \operatorname{sign}\left(P_{i}\right) \operatorname{sign}\left(Q_{i}\right) \operatorname{det}\left(U_{i}\right)\right) \operatorname{det}(S) .
$$

Let $J \in \mathbb{R}^{m \times n}$ be obtained from $I_{n}$ after deleting its block rows $I_{s_{1}}, I_{s_{2}}, \cdots, I_{s_{q}}$, and $U \in \mathbb{C}^{n \times m}$ from $\mathrm{T}$ after deleting its block columns $I_{s_{1}}, I_{s_{2}}, \cdots, I_{s_{q}}$, where $m=l$ when $\mathrm{q}=2$ and $m=n-\left(\sum_{i=1}^{q} s_{i}\right)=\sum_{i=2}^{q}\left(l_{i-1}+r_{i}\right)$ when $\mathrm{q}>2$. Then it can be shown that $T=U J$, so that

$$
S=I_{n}+U J .
$$

The matrix $\mathrm{J}$ is a rectriction/extension matrix; when it post-multiplies a matrix, it extends it and when it pre-multiplies a matrix, it restricts it by selecting some of its block rows. For example if $V_{i}$ and $W_{i}$ are defined by equations (12) and (14), then $J U$ selects the block entries $V_{i}^{t}, V_{i}^{b}, W_{i}^{t}$ and $W_{i}^{b}$ from the matrix $U$.

Let us denote:

$$
\hat{S}=I_{m}+J U .
$$

It follows from Proposition (3.1) and from equations (18) and (19) that the determinant of $S$ and the inverse of $S$ can be computed at reduced cost from $\hat{S}$ as shown in the following proposition:

Proposition 4.2 If $S=I+U J, U \in \mathbb{C}^{n \times m}, J \in \mathbb{C}^{m \times n}$, with $m \leq n$, then

$$
\operatorname{det}(S)=\operatorname{det}(\hat{S})
$$

and

$$
S^{-1}=I_{n}-U \hat{S}^{-1} J
$$


Proof. The second identity is a direct application of the Sherman-Morison-Woodbury formula [5]. The cost for computing the determinant of $S$ is therefore reduced since the dimension of $\hat{S}$ is smaller than that of $S$

After having applied the block odd-even permutation $\Pi$ on both sides of $\hat{S}$, we get a nice pattern for $\hat{S}$ :

$$
\tilde{S}=\Pi^{T} \hat{S} \Pi=\left(\begin{array}{cccc|cccc}
I_{r_{2}} & & & & V_{1}^{b} & & & \\
W_{2}^{b} & I_{r_{3}} & & & & V_{2}^{b} & & \\
& \ddots & \ddots & & & & \ddots & \\
& & W_{q-1}^{b} & I_{r_{q}} & & & & V_{q-1}^{b} \\
\hline W_{2}^{t} & & & & I_{l_{1}} & V_{2}^{t} & & \\
& \ddots & & & & \ddots & \ddots & \\
& & \ddots & & & & \ddots & V_{q-1}^{t} \\
& & & W_{q}^{t} & & & & I_{l_{q-1}}
\end{array}\right) \in \mathbb{C}^{m \times m} .
$$

Thus

$$
\operatorname{det}(S)=\operatorname{det}(\hat{S})=\operatorname{det}(\tilde{S})
$$

\section{Special Case with only two blocks}

We consider the special case of a two-block tridiagonal matrix because the formulas in that case simplify. Let the matrix $A \in \mathbb{C}^{n \times n}$ be defined by:

$$
A=\left(\begin{array}{cc}
A_{1} & A_{12} \\
A_{21} & A_{2}
\end{array}\right)
$$

where $A_{1} \in \mathbb{C}^{n_{1} \times n_{1}}, A_{2} \in \mathbb{C}^{n_{2} \times n_{2}}\left(n=n_{1}+n_{2}\right)$, and where $A_{12}$ and $A_{21}$ are corner matrices defined by:

$$
A_{12}=\left(\begin{array}{cc}
0 & 0 \\
B & 0
\end{array}\right), \text { and } A_{21}=\left(\begin{array}{cc}
0 & C \\
0 & 0
\end{array}\right),
$$

with $B \in \mathbb{C}^{b \times l}$, and $C \in \mathbb{C}^{c \times r}, b \leq n_{1}, c \leq n_{2}, l \leq n_{1}$ and $r \leq n_{2}$. Two cases will be considered: the case where the LU factorization is carried out with left and right permutations, and the case where the LU factorization is carried out without right permutation.

\subsection{Case of two-block with sparse LU factorization with left and right permutation}

For the case of two-block, with the sparse LU factorization using left and right permutation, $P_{1} A_{1} Q_{1}=L_{1} U_{1}$ and $P_{2} A_{2} Q_{2}=L_{2} U_{2}$ we have:

$$
\begin{aligned}
S & =\left(\begin{array}{cccc}
I_{n_{1}-r} & 0 & V_{1}^{\prime} & 0 \\
0 & I_{r} & V_{1}^{b} & 0 \\
0 & W_{2}^{t} & I_{l} & 0 \\
0 & W_{2}^{\prime} & 0 & I_{n_{2}-l}
\end{array}\right) \\
& =I_{n}+U J \text { and } \hat{S}=I_{m}+J U,
\end{aligned}
$$


with

where $m=l$,

$$
\begin{array}{r}
V_{1} \in \mathbb{C}^{n_{1} \times l}=\left|\begin{array}{c|c}
V_{1}^{\prime} & n_{1}-r \\
V_{1}^{b} & r
\end{array}\right| Q_{1} U_{1}^{-1} L_{1}^{-1} P_{1}\left(\begin{array}{c}
0 \\
B
\end{array}\right) \\
W_{2} \in \mathbb{C}^{n_{2} \times r}=\mid \begin{array}{c|c}
W_{2}^{t} & l \\
W_{2}^{\prime} & n_{2}-l
\end{array}=Q_{2} U_{2}^{-1} L_{2}^{-1} P_{2}\left(\begin{array}{c}
C \\
0
\end{array}\right)
\end{array}
$$

$$
U=\left(\begin{array}{cc}
0 & V_{1}^{\prime} \\
0 & V_{1}^{b} \\
W_{2}^{t} & 0 \\
W_{2}^{\prime} & 0
\end{array}\right) \in \mathbb{C}^{n \times m}, J=\left(\begin{array}{cccc}
0 & I_{r} & 0 & 0 \\
0 & 0 & I_{l} & 0
\end{array}\right) \in \mathbb{C}^{m \times n} .
$$

Therefore

$$
\hat{S}=\left(\begin{array}{cc}
I_{r} & V_{1}^{b} \\
W_{2}^{t} & I_{l}
\end{array}\right)=\left(\begin{array}{cc}
I_{r} & 0 \\
W_{2}^{t} & I_{l}
\end{array}\right)\left(\begin{array}{cc}
I_{r} & V_{1}^{b} \\
0 & Z
\end{array}\right),
$$

in which $Z=I-W_{2}^{t} V_{1}^{b} \in \mathbb{C}^{l \times l}$.

Thus we obtain the following proposition which follows straight from Proposition 4.2 when $\mathrm{q}=2$ :

Proposition 5.1 If $A \in \mathbb{C}^{n \times n}$, can be written in the form

$$
A=\left(\begin{array}{cc}
A_{1} & A_{12} \\
A_{21} & A_{2}
\end{array}\right),
$$

where $A_{1} \in \mathbb{C}^{n_{1} \times n_{1}}, A_{2} \in \mathbb{C}^{n_{2} \times n_{2}}\left(n=n_{1}+n_{2}\right)$, and where $A_{12}$ and $A_{21}$ are corner matrices defined by:

$$
A_{12}=\left(\begin{array}{cc}
0 & 0 \\
B & 0
\end{array}\right) \text {, and } A_{21}=\left(\begin{array}{cc}
0 & C \\
0 & 0
\end{array}\right),
$$

with $B \in \mathbb{C}^{b \times l}$, and $C \in \mathbb{C}^{c \times r}, b \leq n_{1}, c \leq n_{2}, l \leq n_{1}$ and $r \leq n_{2}$.

Let $P_{1} A_{1} Q_{1}=L_{1} U_{1}, P_{2} A_{2} Q_{2}=L_{1} U_{2}$ be the sparse $L U$ factorization of $A_{1}$ and $A_{2}$. Let: $P=\operatorname{diag}\left(P_{1}, P_{2}\right), Q=\operatorname{diag}\left(Q_{1}, Q_{2}\right), L=\operatorname{diag}\left(L_{1}, L_{2}\right), U=\operatorname{diag}\left(U_{1}, L_{2}\right)$, then

$$
\operatorname{det}(A)=\operatorname{det}\left(P_{1}\right) \operatorname{det}\left(P_{2}\right) \operatorname{det}\left(Q_{1}\right) \operatorname{det}\left(Q_{2}\right) \operatorname{det}\left(U_{1}\right) \operatorname{det}\left(U_{2}\right) \operatorname{det}\left(I_{l}+W_{2}^{t} V_{1}^{b}\right)
$$

where

and

$$
V_{1}^{b}=\left[\begin{array}{ll}
0 & I_{r}
\end{array}\right] Q_{1} U_{1}^{-1} L_{1}^{-1} P_{1}\left(\begin{array}{c}
0 \\
B
\end{array}\right)
$$

$$
W_{2}^{t}=\left[\begin{array}{ll}
I_{l} & 0
\end{array}\right] Q_{2} U_{2}^{-1} L_{2}^{-1} P_{2}\left(\begin{array}{c}
C \\
0
\end{array}\right) .
$$

Details on the computation of $W_{2}^{t} V_{1}^{b}$ :

Since $V_{1}^{b}=\left[\begin{array}{cc}0 & I_{r}\end{array}\right] V_{1}$ and $W_{2}^{t}=\left[\begin{array}{ll}I_{l} & 0\end{array}\right] W_{2}$, it follows that $W_{2}^{t} V_{1}^{b}$ can be written as:

$$
W_{2}^{t} V_{1}^{b}=\left[\begin{array}{ll}
I_{l} & 0
\end{array}\right] Q_{2} U_{2}^{-1} L_{2}^{-1} P_{2}\left(\begin{array}{c}
C \\
0
\end{array}\right)\left[\begin{array}{ll}
0 & I_{r}
\end{array}\right] Q_{1} U_{1}^{-1} L_{1}^{-1} P_{1}\left(\begin{array}{c}
0 \\
B
\end{array}\right) .
$$

Thus the computation involves $2 r$ triangular systems of dimension $n_{2}$ and $2 l$ triangular systems of dimension $n_{1}$ which can be solved in parallel, and a matrix multiplication of dimension $(l \times r) \times(r \times l)$. 


\subsection{Case of two-block with sparse LU factorization without right permutation}

We now consider the case of a two-block, with the sparse LU factorization without right permutation $P_{1} A_{1}=L_{1} U_{1}$ and $P_{2} A_{2}=L_{2} U_{2}$.

If $Q_{1}=I_{n_{1}}, Q_{2}=I_{n_{2}}$, then

$$
\begin{aligned}
V_{1}^{b} & =\left[\begin{array}{ll}
0 & I_{r}
\end{array}\right] V_{1}, \\
& =\left[\begin{array}{ll}
0 & I_{r}
\end{array}\right] U_{1}^{-1} L_{1}^{-1} P_{1}\left(\begin{array}{c}
0 \\
B
\end{array}\right), \\
& =\left(U_{1}^{-1}\right)^{22}\left[\begin{array}{ll}
0 & I_{r}
\end{array}\right] L_{1}^{-1} P_{1}\left(\begin{array}{c}
0 \\
B
\end{array}\right), \\
W_{2}^{t} & =\left[\begin{array}{ll}
I_{l} & 0
\end{array}\right] W_{2}, \\
& =\left[\begin{array}{ll}
I_{l} & 0
\end{array}\right] U_{2}^{-1} L_{2}^{-1} P_{2}\left(\begin{array}{c}
C \\
0
\end{array}\right), \\
& =\left[\begin{array}{ll}
\left(U_{2}^{-1}\right)^{11} & \left(U_{2}^{-1}\right)^{12}
\end{array}\right] L_{2}^{-1} P_{2}\left(\begin{array}{c}
C \\
0
\end{array}\right),
\end{aligned}
$$

where $\left(U_{2}^{-1}\right)^{11} \in \mathbb{C}^{\left(n_{2}-r\right) \times\left(n_{2}-r\right)},\left(U_{2}^{-1}\right)^{12} \in \mathbb{C}^{\left(n_{2}-r\right) \times r}$, and $\left(U_{2}^{-1}\right)^{22} \in \mathbb{C}^{r \times r}$. Details on the computation of $W_{2}^{t} V_{1}^{b}$ :

From equations (24) and (27), it follows that the computation of $W_{2}^{t}$ involves solving $2 r$ triangular systems of order $n_{2}$ while the computation of $V_{1}^{b}$ involves less operations than in the previous case: $r$ triangular systems of order $l$ are solved instead of 1 triangular systems of order $n_{1}$. These systems can be solved in parallel. The solution of these system is followed by a $(l \times r) \times(r \times l)$ matrix multiplication. The parallel algorithm corresponding to this case is sketched in Algorithm 2.

\section{Parallel algorithms}

We now describe the parallel algorithms for the computation of the determinant of a q-block tridiagonal matrix A. The algorithm for the case of q-block with sparse LU factorization using row and column interchanges is sketched in Algorithm 1 and for the case of two-block without column interchange in Algorithm 2. We have assumed that local data needed by each processor have been provided in a preprocessing step. The factorization of $\tilde{S}$ is carried out by one processor. This of course penalizes the efficiency of the algorithm, as the size of $\tilde{S}$ increases with the number of blocks, especially when the spikes have large bandwidth.

\section{Numerical tests}

Numerical tests were conducted on a desktop, equipped with two processors, each with 6 cores Intel(R) Xeon(R) ; clock : 3.47GHz; RAM: 48GB. The parallel code was 


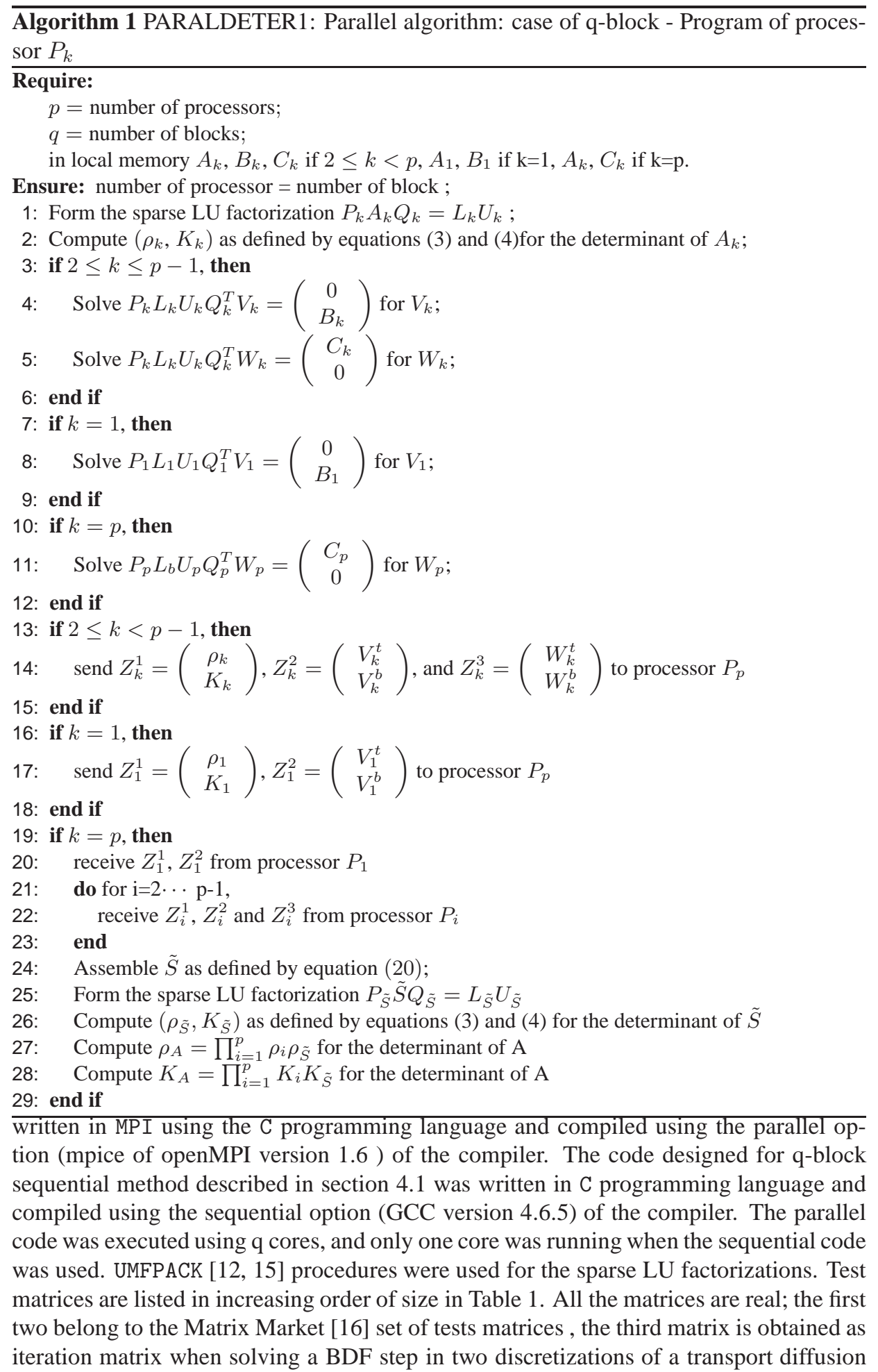




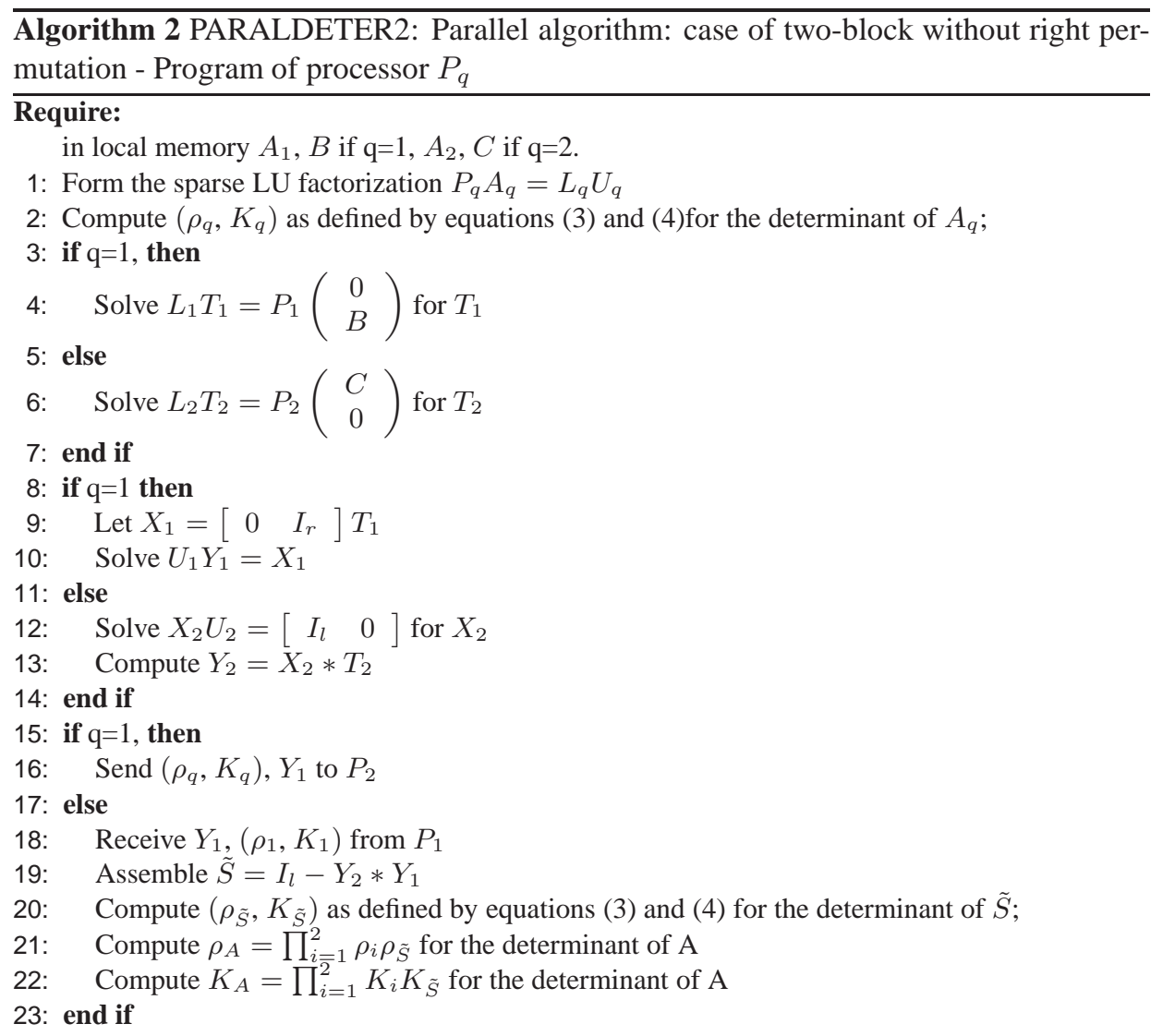

process, the fourth and the fifth matrices were generated randomly using MATLAB function sprand and the last matrix derives from the discretization on the Poisson Equation 2D.

\subsection{Efficiency of the q-block parallel method}

In this section we present the results for the q-block parallel method. Test results show that the efficiency of the parallel algorithm increases until the cost of computing the determinant of $\tilde{S}$, which in our case is carried by one processor, becomes dominant w.r.t the cost for handling one block; several factors may contribute to this situation among which: the size of $\widetilde{S}$, its fill-in and its conditioning. Let $\hat{K}$ denote the number of blocks for which the maximum efficiency is achieved and $\tilde{K}$ the number of blocks for which $\mathrm{m}$ the size of $\tilde{S}$ is equal to average block size i.e. $m=n / \tilde{K}$. For the matrix of Figure 1, Table $2, \hat{K}=6$ while $\tilde{K}=5$ and $\hat{K}$ is slightly larger than $\tilde{K}$; the same observation for the matrix of Figure 2, Table 3 where $\hat{K}=10$ and $\tilde{K}=8$. For the matrices of Figure 3, Table 4 and Figure 6 , Table $7, \hat{K}=7$ and $\hat{K}=9$ respectively, but in both cases $\tilde{K}>12$; the fill-in and conditioning factors may have significantly influenced the cost for handling $\tilde{S}$; it is worth mentioning that in UMFPACK, the LU factorization algorithm seeks to minimize fill-in and to guarantee numerical stability. Finally for matrices of Figure 4, Table 5 and Figure 5, Table 6, both $\hat{K}$ and $\tilde{K}$ are greater than 12 . 


\subsection{Efficiency of the q-block sequential method}

Test results show that the q-block sequential method is faster when $\mathrm{n}$ and $\mathrm{k}$ are large. As shown by the speedup curves of Figure 7 and Figure 8, in some cases the sequential q-block method performs even better than the q-block parallel method, especially when the number of blocks is small. For the matrices of Figure 1 and Figure 6, the sequential algorithm performs better when $k<4$ and for the matrix of Figure 1 when $k \geq 10$.

\subsection{Consequences from the efficiency of the proposed algorithms}

From the test results obtained for the set of matrices, the following consequences could be drawn:

- the q-block sequential method allows one to consider on one processor large matrices that cannot be handled in one unit.

- The q-block parallel method is efficient on $\hat{k}$ processors when $\hat{k}$ is such that the average block size $\hat{n}=\frac{n}{\hat{k}}$ is not small when compared to $\mathrm{m}$, the size of $\tilde{R}$.

Table 1. Characteristics of the test matrices (Name: Matrix Market name (if from Matrix Market); $n$ : order of the matrix ; $m$ : order of $\tilde{S}$ for 12 blocks; NZ/line: number of non zeros elements par line.)

\begin{tabular}{l|r|l|l|r} 
Name & $n$ & NZ/line & Type & $m$ \\
\hline ER40R5000 & 17,281 & 32.03 & Real non-symmetric & 13,002 \\
S3DKQ4M2 & 90,449 & 48.95 & Real symmetric & 16,980 \\
ITERATION MATRIX & 300,000 & 7.4 & Real non-symmetric & 12,280 \\
RANDOM MATRIX & 500,000 & 30.99 & Real non-symmetric & 330 \\
RANDOM MATRIX & $1,000,000$ & 40.99 & Real non-symmetric & 440 \\
POISSON MATRIX & $1,000,000$ & 4.99 & Real symmetric & 15,694
\end{tabular}

Table 2. Run time table for Matrix Market matrix e40r5000 where $n=17281$ NBlock: number of blocks; PTime: parallel time; STime: sequential time

\begin{tabular}{|c|c|c|c|c|c|c|c|c|c|c|c|}
\hline NBblock & 2 & 3 & 4 & 5 & 6 & 7 & 8 & 9 & 10 & 11 & 12 \\
\hline STime & 11.08 & 15.76 & 18.14 & 19.97 & 21.16 & 22.56 & 23.77 & 24.12 & 25.58 & 24.76 & 27.00 \\
\hline PTime & 25.03 & 19.69 & 14.45 & 13.54 & 12.92 & 13.96 & 16.40 & 19.54 & 26.80 & 31.67 & 38.97 \\
\hline
\end{tabular}


Table 3. Run time table for Matrix Market matrix s3dkq4m2 where $n=90449$ NBlock: number of blocks; PTime: parallel time; STime: sequential time

\begin{tabular}{|c|c|c|c|c|c|c|c|c|c|c|c|}
\hline NBblock & 2 & 3 & 4 & 5 & 6 & 7 & 8 & 9 & 10 & 11 & 12 \\
\hline STime & 30.68 & 38.56 & 38.50 & 32.68 & 34.40 & 31.51 & 32.56 & 33.93 & 31.45 & 32.44 & 33.27 \\
\hline PTime & 49.76 & 32.34 & 33.01 & 30.99 & 25.60 & 24.05 & 22.65 & 21.02 & 20.51 & 21.98 & 21.54 \\
\hline
\end{tabular}

Table 4. Run time table for the Iteration matrix $A 300000$ where $n=300000$. NBlock: number of blocks; PTime: parallel time; STime: sequential time

\begin{tabular}{|c|c|c|c|c|c|c|c|c|c|c|c|}
\hline NBblock & 2 & 3 & 4 & 5 & 6 & 7 & 8 & 9 & 10 & 11 & 12 \\
\hline STime & 116.56 & 150.43 & 130.95 & 126.01 & 125.64 & 122.06 & 117.83 & 116.12 & 113.31 & 109.85 & 106.35 \\
\hline PTime & 112.62 & 123.36 & 91.27 & 82.92 & 75.03 & 70.47 & 70.85 & 77.26 & 76.25 & 75.69 & 82.62 \\
\hline
\end{tabular}

Table 5. Run time table for random matrix LB500000 where $n=500000$. NBlock: number of blocks; PTime: parallel time; STime: sequential time

\begin{tabular}{|c|c|c|c|c|c|c|c|c|c|c|c|}
\hline NBblock & 2 & 3 & 4 & 5 & 6 & 7 & 8 & 9 & 10 & 11 & 12 \\
\hline STime & 72.79 & 80.44 & 71.89 & 56.60 & 38.12 & 12.04 & 10.69 & 10.85 & 10.85 & 10.99 & 11.03 \\
\hline PTime & 86.70 & 85.66 & 51.55 & 30.21 & 15.72 & 3.82 & 3.06 & 2.81 & 2.73 & 2.72 & 2.59 \\
\hline
\end{tabular}

Table 6. Run time table the random matrix LBmillion where $n=1000000$. NBlock: number of blocks; PTime: parallel time; STime: sequential time

\begin{tabular}{|c|c|c|c|c|c|c|c|c|c|c|c|}
\hline NBblock & 2 & 3 & 4 & 5 & 6 & 7 & 8 & 9 & 10 & 11 & 12 \\
\hline STime & 240.22 & 257.82 & 222.33 & 165.60 & 98.19 & 34.12 & 34.52 & 35.04 & 35.39 & 35.76 & 36.07 \\
\hline PTime & 294.35 & 271.37 & 147.91 & 70.38 & 25.23 & 10.73 & 9.73 & 9.52 & 8.89 & 8.85 & 8.40 \\
\hline
\end{tabular}

Table 7. Run time table for the 3D Poisson Matrix were $n=1000$ 000. NBlock: number of blocks; PTime: parallel time; STime: sequential time

\begin{tabular}{|c|c|c|c|c|c|c|c|c|c|c|c|}
\hline NBblock & 2 & 3 & 4 & 5 & 6 & 7 & 8 & 9 & 10 & 11 & 12 \\
\hline STime & 99.86 & 93.36 & 103.26 & 98.95 & 98.93 & 98.07 & 97.02 & 94.35 & 94.94 & 92.33 & 91.92 \\
\hline PTime & 1323.74 & 270.36 & 122.30 & 54.73 & 54.95 & 51.81 & 50.15 & 46.38 & 49.42 & 48.69 & 53.30 \\
\hline
\end{tabular}




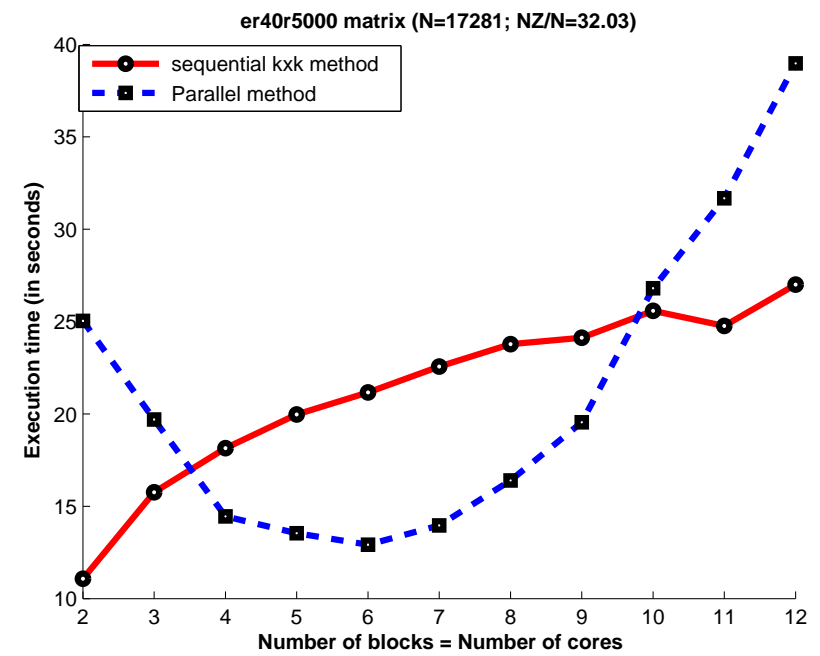

Figure 1. Run time curve for the Matrix Market matrix e40r5000 where $n=17281$. Parallel case: Algorithm 1 used. Sequential case: q-block sequential method used with one core

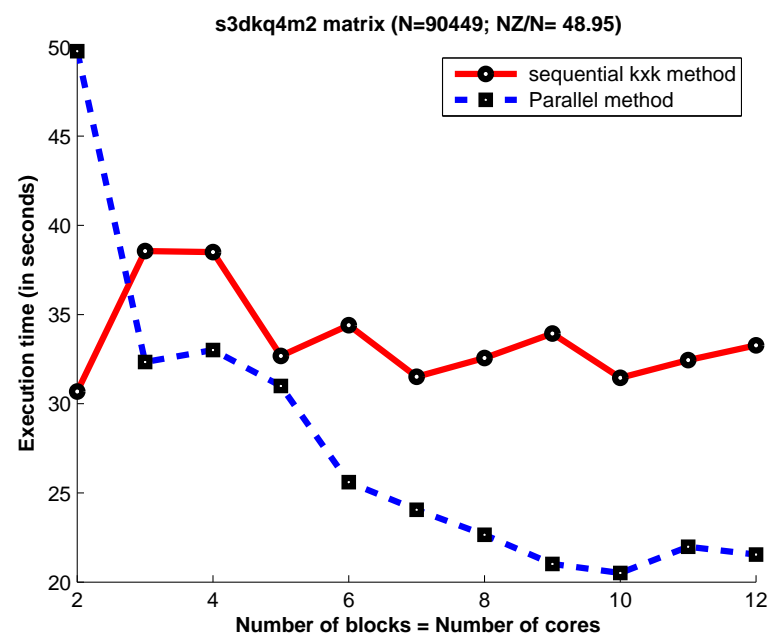

Figure 2. Run time curve for the Matrix Market matrix s3dkq4m2 where $n=90449$. Parallel case: Algorithm 1 used. Sequential case: q-block sequential method used with one core 


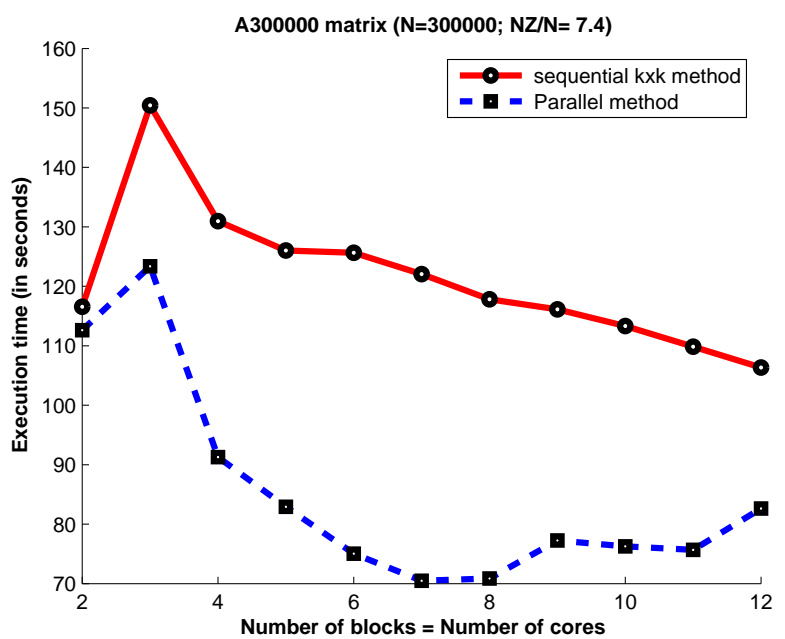

Figure 3. Run time curve for the Iteration matrix A300000 where $n=300000$. Parallel case: Algorithm 1 used. Sequential case: q-block sequential method used with one core

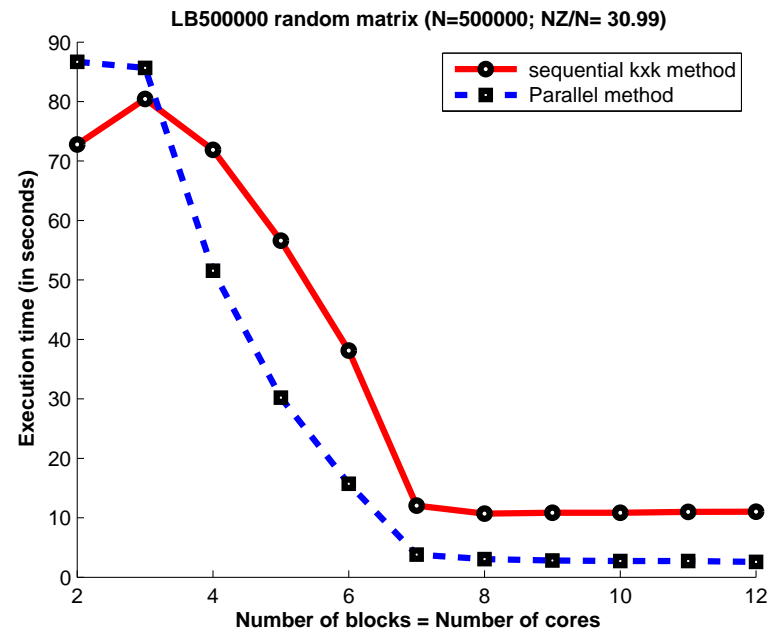

Figure 4. Run time curve for the random matrix LB500000 where $n=500000$. Parallel case: Algorithm 1 used. Sequential case: q-block sequential method used with one core 


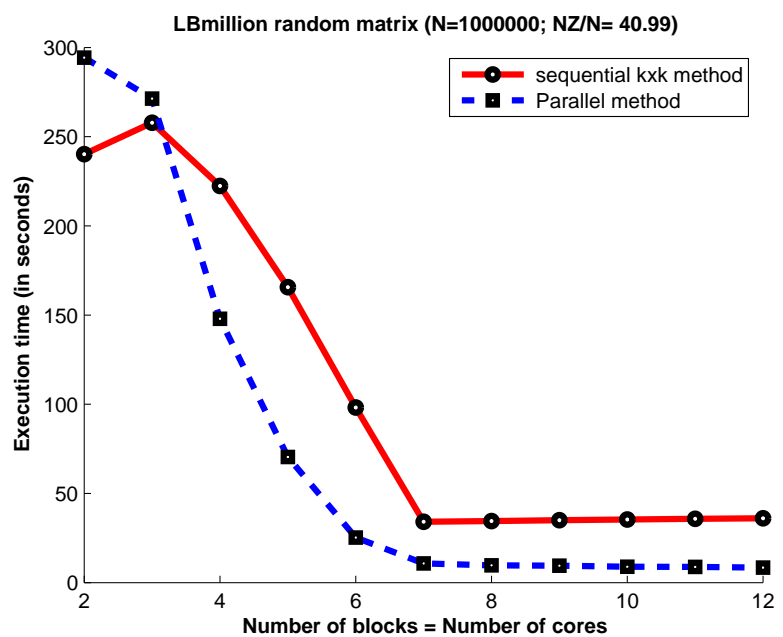

Figure 5. Run time curve for the random matrix LBmillion where $n=1000$ 000. Parallel case: Algorithm 1 used. Sequential case: q-block sequential method used with one core.

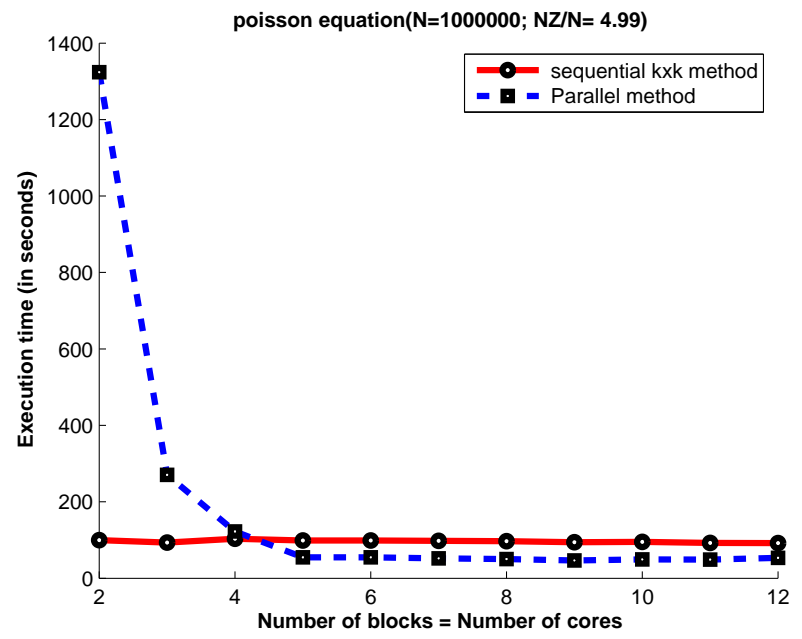

Figure 6. Run time curve for the $3 D$ Poisson Matrix where $n=1000000$. Parallel case: Algorithm 1 used. Sequential case: q-block sequential method used with one core. 


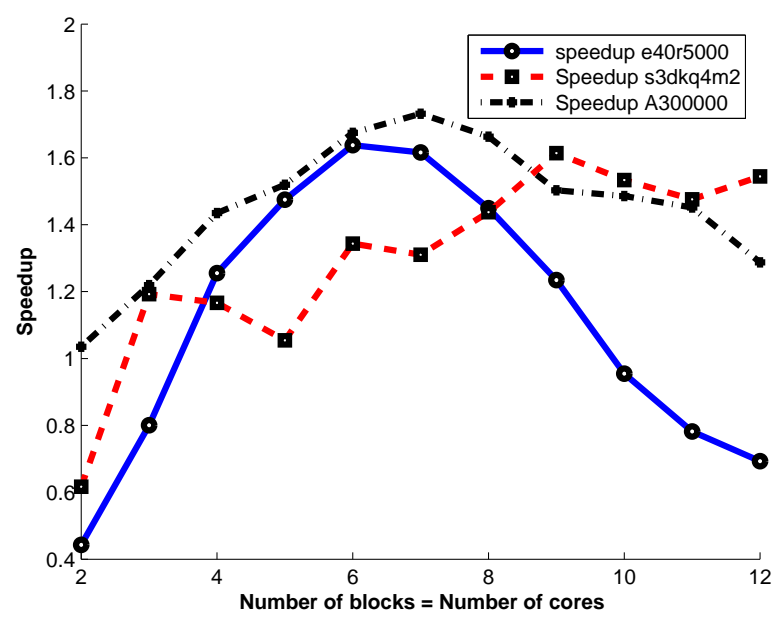

Figure 7. Speedup curves for the Matrix Market matrix e40r5000 where $n=17281$, the Matrix Market matrix s3dkq4m2 where $n=90449$ and the Iteration matrix A300000 where $n=300000$.

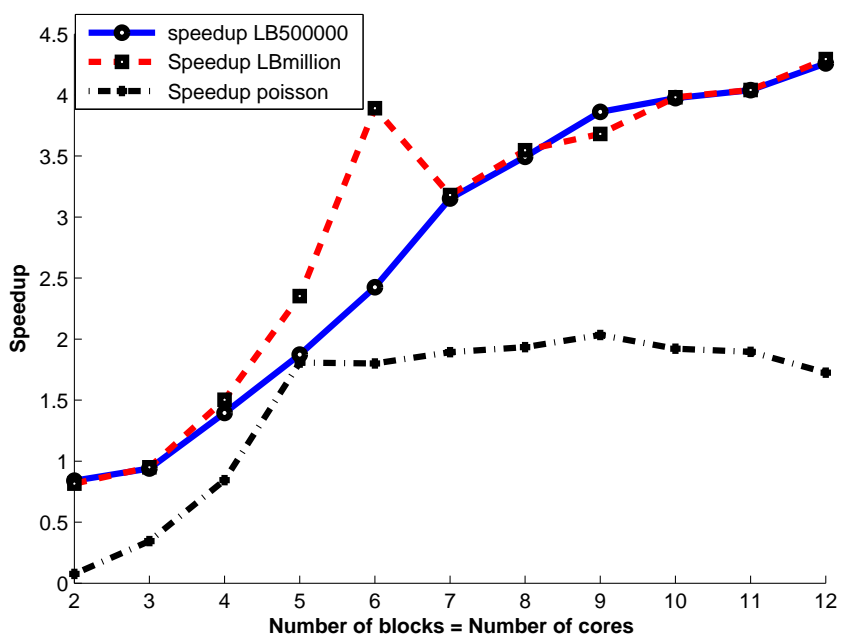

Figure 8. Speedup curves for the random matrix LB500000 where $n=500000$, the random matrix LBmillion where $n=1000000$ and the 3D Poisson Matrix where $n=1000$ 000 . 


\section{Conclusions}

We have described some efficient methods for computing the determinant of large block structured matrices. Test results indicate that the q-block sequential method is efficient when using one processor on large matrices. The q-block parallel method is efficient when $\mathrm{k}$ the number of processors is such that the average block size $\frac{n}{k}$ is not small compared to the size of $\tilde{R}$. Tests conducted on a set of matrices confirm the efficiency of the proposed algorithms; the efficiency of the parallel algorithm, however is penalized when the spikes have large widths and when be number of blocks increases; this is due to the fact that in our current algorithm, the computation of the determinant of $\tilde{S}$ is carried by one processor; i.e. sequentially and Amdahl's law applies. In future work, we intend to implement a parallel version of the LU factorization of $\tilde{S}$.

\section{Acknowledgement}

The authors would like to thank Bernard Philippe for all his suggestions and assistance. This work was supported in part by the projet LIRIMA through the projet team MOMAPLI, and was carried out during the authors visits within the research team SAGE at the INRIA Rennes-Bretagne Atlantique center.

\section{References}

[1] G. A. Atenekeng-Kahou, L. Grigori, and M. Sosonkina. A partitioning algorithm for blockdiagonal matrices with overlap. Parallel Computing, 34:332 - 344, 2008.

[2], O. Bertrand and B. Philippe. Counting the eigenvalues surrounded by a closed curve, Sib. Zh. Ind. Mat.,4:73 - 94, 2001.

[3] E. Kamgnia and B. Philippe. Counting eigenvalues in domains of the complex field. Electronic Transactions on Numerical Analysis.,40:1 - 16, 2013.

[4] D. Bindel Bounds and error estimates for nonlinear eigenvalue problems, Berkeley Applied Mathematical Seminar, October 2008

[5] G. Golub and C. V. Loan Matrix Computation, p51; The Johns Hopkins University Press, Second Edition, 1989.

[6] G. Karypis and V. Kumar. METIS - unstructured graph partitioning and sparse matrix ordering system, version 2.0. University of Minnesota, CS Dept, Technical report, 1995.

[7] E. Polizzi and A. Sameh A parallel hybrid banded systems solver: the SPIKE algorithm Parallel Computing, 32:177-194, 2006.

[8] Maeda, Yasuyuki, Futamura, Yasunori, Sakurai and Tetsuya Stochastic estimation method of eigenvalue density for nonlinear eigenvalue problem on the complex plane JSIAM Letters , 3:61 $-64,2011$

[9] C. Meyer Matrix Analysis and Applied Linear Algebra SIAM, pp 483, 2000

[10] SuperLU; X. Sherry, L. J. Demmel, J. Gilbert, L. Grigori, M. Shao, and I. Yamazaki. SuperLU is a general purpose bibrary for the direct solution of large, sparse, nonsymmetric systems of 
$92 \quad$ A R I M A - Volume $17-2014$

linear equations on high performance machines http://crd-legacy.lbl.gov/xiaoye/SuperLU

[11] MUMPS; Multifrontal Massively Parallel sparse direct Solver http://graal.enslyon.fr/MUMPS/

[12] UMFPACK; Unsymmetric Multifrontal Sparse LU Factorization Package; http://www.cise.ufl.edu/research/sparse/umfpack/

[13] T. A. Davis Algorithm 832: UMFPACK, an unsymmetric-pattern multifrontal method ACM Transactions on Mathematical Software, 30:2, 196 - 199, 2004.

[14] T. A. Davis and I. S. Duff A combined unifrontal/multifrontal method for unsymmetric sparse matrices, ACM Transactions on Mathematical Software, 25.1, 1 - 19, 1999.

[15] T. A. Davis and I. S. Duff, An unsymmetric-pattern multifrontal method for sparse LU factorization SIAM Journal on Matrix Analysis and Applications, 18.1, 140 - 158, 1997.

[16], Matrix Market, Service of the Mathematical and Computational Sciences Division / Information Technology Laboratory / National Institute of Standards and Technology, http://math.nist.gov/MatrixMarket/. 